\title{
New trends in MRI of cartilage: Advances and limitations in small animal studies
}

\author{
Jean-Christophe Goebel ${ }^{\mathrm{a}}$, Astrid Pinzano ${ }^{\mathrm{b}}$, Denis Grenier ${ }^{\mathrm{a}}$, Anne-Laure Perrier ${ }^{\mathrm{a}}$, \\ Christel Henrionnet ${ }^{\mathrm{b}}$, Laurent Galois ${ }^{\mathrm{b}}$, Pierre Gillet ${ }^{\mathrm{b}}$ and Olivier Beuf ${ }^{\mathrm{a}, *}$ \\ ${ }^{\text {a } C R E A T I S-L R M N, ~ C N R S ~ U M R ~ 5220, ~ I n s e r m ~ U 630, ~ I N S A-L y o n, ~ U n i v e r s i t e ́ ~ L y o n ~ 1, ~ V i l l e u r b a n n e, ~}$ \\ France \\ ${ }^{\mathrm{b}}$ PPIA, CNRS UMR 7561, Nancy Université, Vandoeuvre-lès-Nancy, France
}

\begin{abstract}
Due to the actual interest for bioengineering in the osteoarthritis (OA) healing context, researchers need accurate qualitative and quantitative methodologies to evaluate in vivo the integration and functionality of their cartilage-like biomaterials. As in clinical diagnostic strategies, advances in Magnetic Resonance Imaging (MRI) seem promising for non-vulnerant assessments of articular cartilage bio-architecture and morphology in small animal models. These experimental models are commonly used to monitor the physiopathology of OA and to evaluate therapeutic responses mediated by chondroprotective drugs or tissue engineering. Nowadays, the application of MR protocols to in vivo small animal cartilage imaging is achievable with the development of high magnetic fields and the adaptation of methodologies to reach the required spatial resolution and contrast. The purpose of this article is to summarize these current MRI strategies used for in vivo small animal articular cartilage assessments.
\end{abstract}

Keywords: MRI, cartilage, osteoarthritis, animal models, bioengineering, extracellular matrix

\section{Introduction}

Given superior tissue contrast and sensitivity to tissue composition, Magnetic Resonance Imaging (MRI) has a tremendous potential in the study of cartilage repair. Indeed, it may (a) allow one to monitor degenerative changes in the joint (early diagnosis), (b) estimate the size, nature and location of lesions before surgery and (c) evaluate the quality and success of tissue repair processes after surgical treatment. However, despite the use of still higher magnetic fields (7 Teslas and more), in vivo MRI can hardly achieve today the same degree of spatial resolution, proportionally, on small animals compared to humans. Nevertheless, different studies have yet shown that qualitative assessment of degenerative joint disease, derived directly from MR images, was reliable. The capability to show pathologic changes throughout the time course of the disease from three-dimensional (3D) datasets has also been demonstrated [1]. However, in vivo quantitative imaging for an accurate determination of cartilage thickness and volume is challenging due to small size of rodent joints. Dedicated instrumental and methodological developments have to be performed to achieve a reproducible and accurate measurement of both thickness and volume on experimental guinea pig [2,3] and rat model of osteoarthritis (OA) [4]. Beyond morphology, specific MRI techniques have the potential to indirectly give access to the composition of the cartilage matrix ultra structure. The most promising techniques include the transverse relaxation time T2,

\footnotetext{
*Address for correspondence: Olivier Beuf, CREATIS-LRMN, CNRS UMR 5220, Domaine Scientifique de la Doua, Bât. ESCPE, 3, rue Victor Grignard, F-69616 Villeurbanne Cedex, France. E-mail: olivier.beuf@univ-lyon1.fr.
} 
the longitudinal relaxation time T1 in the presence of gadolinium (delayed gadolinium-enhanced MRI of cartilage (dGEMRIC) method), diffusion-weighted imaging and more recently T1rho. The interest of each technique for cartilage physiopathology imaging will be discussed regarding small animal applications.

\section{General recommendations for in vivo small animal imaging}

Prior to MRI acquisitions, the examination of small animal models can require development of dedicated apparatus, such as specific coils to improve signal-to-noise and contrast-to-noise ratios [5]. In any cases, the whole emission-reception chain must be matched and tuned to the frequency fixed by the magnetic field strength.

Guidelines for animal experimentation have to be followed strictly. Adapted anesthesia protocols as well as total scan time duration must be compatible with animal welfare and required image quality.

\section{Morphological assessments}

A direct first approach to characterize articular cartilage is based on morphological measurements. Under this designation are regrouped typical analyses like whole cartilage volume extraction, thickness and roughness mapping, as well as weight-bearing areas measurement. This type of data is commonly obtained after segmentation of cartilage compartments. In general, cartilage-sensitive sequences such as fat-suppressed three-dimensional gradient echo are well suited for accurate cartilage delineation $[1,4,6]$. Indeed, cartilage appears in hypersignal intensity so contrasting with bone, synovial fluid and other articular constituents such as meniscus or ligaments (Fig. 1). Morphological parameters are then extracted and quantified by dedicated image post-processing $[2,3,7]$. Another approach, derived from clinical studies, can be applied and consists in semi-quantitative scoring methods. In this case, MR images are read by trained operators, and cartilage abnormalities or defects are graded according to a severity-scaled grid $[8,9]$.

Due to limited size of small animal joints, morphological cartilage assessment remains very challenging. Dedicated phased array coil designed for $7 \mathrm{~T}$ considerably improve the SNR as well as the signal uniformity. Both parameters are mandatory to segment and to quantify cartilage morphology (thickness, volume $)$ with good reproducibility. The spatial resolution value achieved with such coil $\left(51 \times 51 \times 94 \mu^{3}\right.$ voxel size) was the highest never been reported in the literature [5]. Moreover, High spatial Resolution MRI (HR-MRI) of both joints performed simultaneously significantly increase throughput analysis.

\section{Transverse relaxation time (T2) mapping}

On a histological point of view, mature articular cartilage can be described as few chondrocytes surrounded by a wide and highly organized extracellular matrix. This matrix is composed by collagen fibers anchored onto the subchondral bone and forming arcs by approaching the articular cartilage surface. In addition, large amounts of proteoglycans are present in the extracellular matrix, and their concentration varies from the deeper to the articular stratum [10,11]. This anisotropic molecular architecture involves 


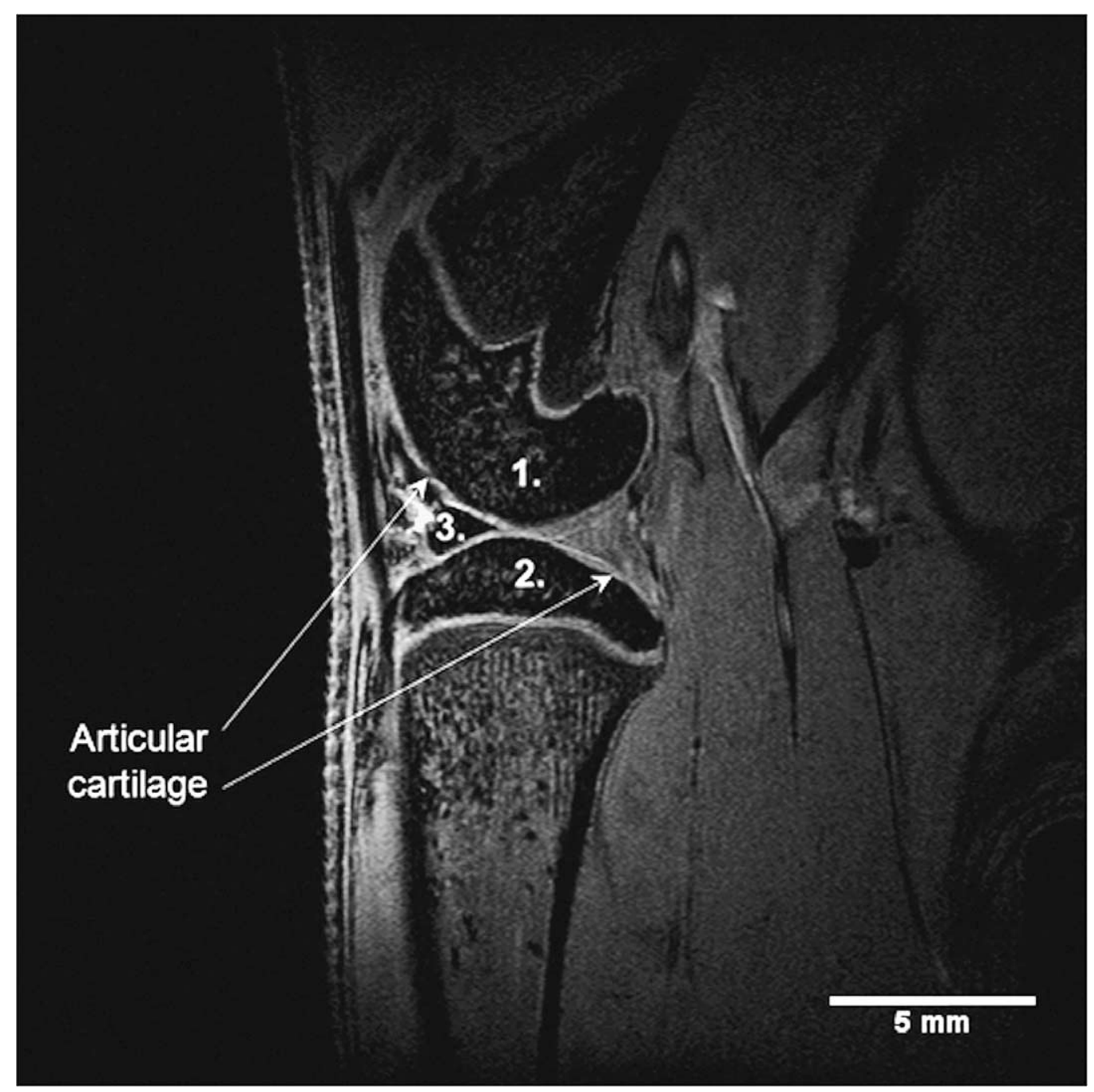

Fig. 1. Rat knee MR image obtained in vivo at $7 \mathrm{~T}$ from a 3D gradient echo sequence with fat suppression acquired with a $51 \times 51 \times 94 \mu^{3}$ voxel size. (1) and (2) correspond to the femoral and tibial epiphysis, respectively, (3) represents the anterior horn of the meniscus. Arrows mention femoral and tibial articular cartilages.

specific water-fibers interactions, leading to stratified and constrained water pools into articular cartilage. These local water contents, related to the fibers biochemistry, can be quantified by T2-value computation.

For that purpose, most of studies report the use of multi-slices multiple spin-echo sequences. Subsequently, cartilage T2-maps are commonly generated using a mono- or dual-exponential curve fitting method, reproducing the MR signal decay thus delivering for each voxel the corresponding T2-value.

As observed in vitro on enzymatically treated patellar cartilage, measurements of articular cartilage $\mathrm{T} 2$ provide an imaging biomarker of structural changes in the collagen network as well as proteoglycans denaturation [12]. In vivo, increased T2-values are consistent with relative augmentation of the water content in a cartilage degeneration model [13]. 


\section{Delayed gadolinium-enhanced MRI of cartilage (dGEMRIC)}

Glycosaminoglycans (GAG), structural polysaccharides of the extracellular matrix, represent a tall density of negative charges within cartilage. These anionic molecules have been reported to be altered in the early stage of cartilage damages [14]. Intravenously administrated gadolinium diethylenetriamine pentaacetate anion (Gd-DTPA ${ }^{2-}$ ) penetrates the cartilage through the articular surface and the subchondral bone. A GAG depletion leads to a loss of negative charges into the extracellular matrix. This anionic attenuation allows the contrast medium to better penetrate cartilage, in proportion of the GAG decrease. Hence, accumulation of contrast agent due to the depletion of GAG in cartilage leads to decreased T1values (contrast enhancement with T1-weighted imaging). Longitudinal relaxation time (T1) mapping, related to Gd-DTPA ${ }^{2-}$ concentration, can therefore be used as a specific measure of the cartilage GAG content [15].

Although dGEMRIC represents an elegant GAG dosing method, it is noteworthy to bring some limitations for an in vivo animal application. In fact, a commonly accepted period of 90 min between GdDTPA $^{2-}$ injection and post-contrast imaging must be respected, in order to allow sufficient time for the contrast agent to diffuse into the cartilage. This one hour and half time must be considered in the study design.

\section{Diffusion-weighted imaging}

Diffusion-weighted imaging (DWI) is another promising strategy delivering informations about the regional anisotropic variations of cartilage ultrastructure [16]. Principle of DWI is based on molecular motion which is influenced by interactions with the cartilage constituents. In other words, it is possible to estimate the extracellular bio-architecture by measuring molecular movements. In vivo application of DWI for small animal actually remains challenging due to long acquisition times using spin-echo sequence or unwanted artifacts and lack of spatial resolution using Echo Planar Imaging (EPI) sequences.

\section{T1rho mapping}

T1rho relaxation time has recently been proposed as a pertinent parameter to measure biochemical variations into articular cartilage. T1rho describes the spin-lattice relaxation in the rotating frame. T1rhomaps bring to light the strong relation between signal intensity and cartilage proteoglycans content [17, 18]. T1rho measurements can be used to probe slow motion interactions between motion-restricted water molecules and their local macromolecular surroundings. Cartilage matrix constitutes a motion-restricted environment to water molecules. In this manner, early osteoarthritic manifestations like modifications in proteoglycans concentration can be detected by altered T1rho values [19].

\section{Cell labeling and tracking}

So far, we mainly told about MRI and its utilizations for matrix evaluation rather than for studies of the cellular component of cartilage. Techniques that would characterize cellular distribution within chondrogenic biomaterials would be of particular interest because cellular distribution would affect the uniformity of matrix deposition within the construct, as well as the overall viability and functionality 
of tissue-engineered cartilage. Development of methodologies such as cell labeling will offer the opportunity to determine whether cells are retained within the scaffold and whether there is a random distribution of cells within the construct. Actually, superparamagnetic iron oxide (SPIO) nanoparticles appear to be good candidates for cell labeling [20]. Intracellular iron particles reduce local magnetic field homogeneity, allowing the cells to be visualized as signal voids using apparent longitudinal relaxation time $\mathrm{T} 2{ }^{*}$-weighted MR images. The application of such methods in the context of bioengineered constructs may be particularly useful as a noninvasive, nondestructive method of monitoring cellular distribution. Ultimately, such methods should permit longitudinal cell tracking over a period of several weeks after seeding [21].

\section{Conclusion}

Actual development of high magnetic fields with dedicated measurement strategies highlight MRI capabilities to directly or indirectly monitor and quantify cartilage physiopathology over crosssectional [22] and longitudinal planes. Current advances allow good expectancy for bioengineering, thus providing non-vulnerant methodologies to evaluate and follow in vivo therapeutical responses after chondrogenic biomaterial grafting.

\section{Acknowledgements}

Jean Christophe GOEBEL's post doc position is granted by ANR CEMABIR *ANR-09-BLAN-015001. We also thank "Conseil Général de Meurthe et Moselle", "Communauté Urbaine du Grand Nancy" and "Conseil Régional" for their financial support.

\section{References}

[1] J.J. Tessier, J. Bowyer, N.J. Brownrigg, I.S. Peers, F.R. Westwood, J.C. Waterton and R.A. Maciewicz, Characterisation of the guinea pig model of osteoarthritis by in vivo three-dimensional magnetic resonance imaging, Osteoarthr. Cartil. 11 (2003), 845-853.

[2] R. Bolbos, H. Benoit-Cattin, J.B. Langlois, A. Chomel, E. Chereul, C. Odet, M. Janier, P. Pastoureau and O. Beuf, Measurement of knee cartilage thickness using MRI: a reproducibility study in a meniscectomized guinea pig model of osteoarthritis, NMR Biomed. 21 (2008), 366-375.

[3] R. Bolbos, H. Benoit-Cattin, J.B. Langlois, A. Chomel, E. Chereul, C. Odet, P. Pastoureau, M. Janier and O. Beuf, Knee cartilage thickness measurements using MRI: a $4 \frac{1}{2}$-month longitudinal study in the meniscectomized guinea pig model of OA, Osteoarthr. Cartil. 15 (2007), 656-665.

[4] J.C. Goebel, R. Bolbos, A. Pinzano, M. Schaeffer, A. Rengle, L. Galois, S. Etienne, P. Netter, D. Loeuille, O. Beuf and P. Gillet, In vivo rat knee cartilage volume measurement using quantitative high resolution MRI (7 T): Feasibility and reproducibility, Biomed. Mater. Eng. 18 (2008), 247-252.

[5] A. Rengle, M. Armenean, R. Bolbos, J.C. Goebel, A. Pinzano-Watrin, H. Saint-Jalmes, P. Gillet and O. Beuf, A dedicated two-channel phased array receiver coil for high resolution MRI of the rat knee cartilage at 7 T, IEEE Trans. Biomed. Eng. 56 (2009), 2891-2897.

[6] P. Faure, B.T. Doan and J.C. Beloeil, In vivo high resolution three-dimensional MRI studies of rat joints at 7 T, NMR Biomed. 16 (2003), 484-493.

[7] P. Pouletaut, J.C. Goebel, A. Pinzano, R. Bolbos, O. Beuf, P. Netter, M.C. Ho Ba Tho and P. Gillet, MRI study of rat cartilage ageing process: knee joint contact sites geometrical assessments, Comput. Methods Biomech. Biomed. Engin. 11(Suppl. 1) (2008), 183-184.

[8] C.G. Peterfy, A. Guermazi, S. Zaim, P.F. Tirman, Y. Miaux, D. White, M. Kothari, Y. Lu, K. Fye, S. Zhao and H.K. Genant, Whole-Organ Magnetic Resonance Imaging Score (WORMS) of the knee in osteoarthritis, Osteoarthr. Cartil. 12 (2004), 177-190. 
[9] G.H. Welsch, L. Zak, T.C. Mamisch, C. Resinger, S. Marlovits and S. Trattnig, Three-dimensional magnetic resonance observation of cartilage repair tissue (MOCART) score assessed with an isotropic three-dimensional true fast imaging with steady-state precession sequence at 3.0 Tesla, Invest. Radiol. 44 (2009), 603-612.

[10] X. Chevalier, Physiopathology of arthrosis. The normal cartilage, Presse Med. 27 (1998), 75-80.

[11] D.R. Jeffrey and I. Watt, Imaging hyaline cartilage, Br. J. Radiol. 76 (2003), 777-787.

[12] A. Watrin-Pinzano, J.P. Ruaud, P. Olivier, L. Grossin, P. Gonord, A. Blum, P. Netter, G. Guillot, P. Gillet and D. Loeuille, Effect of proteoglycan depletion on T2 mapping in rat patellar cartilage, Radiology 234 (2005), 162-170.

[13] M.C. Chou, P.H. Tsai, G.S. Huang, H.S. Lee, C.H. Lee, M.H. Lin, C.Y. Lin and H.W. Chung, Correlation between the MR T2 value at $4.7 \mathrm{~T}$ and relative water content in articular cartilage in experimental osteoarthritis induced by ACL transaction, Osteoarthr. Cartil. 17 (2009), 441-447.

[14] P. Lorenzo, M.T. Bayliss and D. Heinegard, Altered patterns and synthesis of extracellular matrix macromolecules in early osteoarthritis, Matrix Biol. 23 (2004), 381-391.

[15] S. Trattnig, S. Domayer, G.W. Welsch, T. Mosher and F. Eckstein, MR imaging of cartilage and its repair in the knee a review, Eur. Radiol. 19 (2009), 1582-1594.

[16] K.L. Miller, B.A. Hargreaves, G.E. Gold and J.M. Pauly, Steady-state diffusion-weighted imaging of in vivo knee cartilage, Magn. Reson. Med. 51 (2004), 394-398.

[17] R. Stahl, A. Luke, X. Li, J. Carballido-Gamio, C.B. Ma, S. Majumdar and T.M. Link, T1rho, T2 and focal knee cartilage abnormalities in physically active and sedentary healthy subjects versus early OA patients - a 3.0-Tesla MRI study, Eur. Radiol. 19 (2009), 132-143.

[18] N.M. Menezes, M.L. Gray, J.R. Hartke and D. Burstein, T2 and T1rho MRI in articular cartilage systems, Magn. Reson. Med. 51 (2004), 503-509.

[19] J. Lozano, E. Saadat, X. Li, S. Majumdar and C.B. Ma, Magnetic resonance T(1 rho) imaging of osteoarthritis: a rabbit ACL transection model, Magn. Reson. Imaging 27 (2009), 611-616.

[20] E. Farrell, P. Wielopolski, P. Pavljasevic, N. Kops, H. Weinans, M.R. Bernsen and G.J. van Osch, Cell labelling with superparamagnetic iron oxide has no effect on chondrocyte behavior, Osteoarthr. Cartil. 17 (2009), 961-967.

[21] S. Ramaswamy, J.B. Greco, M.C. Uluer, Z. Zhang, K.W. Fishbein and R.G. Spencer, Magnetic resonance imaging of chondrocytes labeled with superparamagnetic iron oxide nanoparticles in tissue-engineered cartilage, Tissue Eng. Part A 15 (2009), 3899-3910.

[22] J.C. Goebel, R. Bolbos, M. Pham, L. Galois, A. Rengle, D. Loeuille, P. Netter, P. Gillet, O. Beuf and A. Watrin-Pinzano, In vivo high-resolution MRI (7T) of femoro-tibial cartilage changes in the rat anterior cruciate ligament transection model of osteoarthritis: a cross-sectional study, Rheumatology 49 (2010), 1654-1664. 